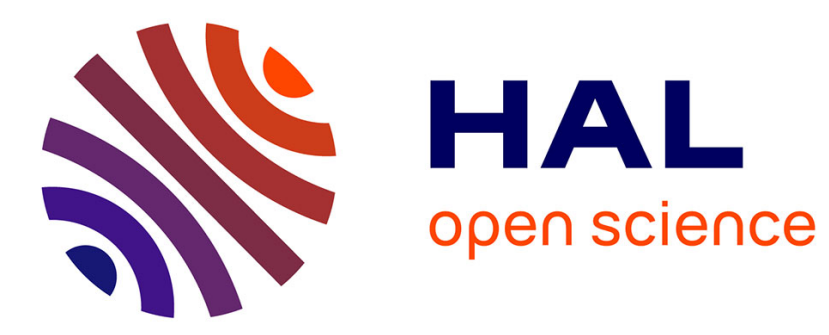

\title{
3D printed PEGDA microstructures for gelatin scaffold integration and neuron differentiation
}

Xiaolong Tu, Lina Wang, Wei Jin, Bin Wang, Yadong Tang, Jian Shi, Zhijun Zhang, Yong Chen

\section{- To cite this version:}

Xiaolong Tu, Lina Wang, Wei Jin, Bin Wang, Yadong Tang, et al.. 3D printed PEGDA microstructures for gelatin scaffold integration and neuron differentiation. Microelectronic Engineering, 2016, 10.1016/j.mee.2016.03.007 . hal-01285501

\section{HAL Id: hal-01285501 \\ https: / hal.sorbonne-universite.fr/hal-01285501}

Submitted on 9 Mar 2016

HAL is a multi-disciplinary open access archive for the deposit and dissemination of scientific research documents, whether they are published or not. The documents may come from teaching and research institutions in France or abroad, or from public or private research centers.
L'archive ouverte pluridisciplinaire HAL, est destinée au dépôt et à la diffusion de documents scientifiques de niveau recherche, publiés ou non, émanant des établissements d'enseignement et de recherche français ou étrangers, des laboratoires publics ou privés. 


\title{
3D printed PEGDA microstructures for gelatin scaffold integration and neuron differentiation
}

\author{
Xiaolong Tu ${ }^{\mathrm{a}}$, Lina Wang ${ }^{\mathrm{b}}$, Wei Jin ${ }^{\mathrm{a}}$, Bin Wang ${ }^{\mathrm{a}}$, Yadong Tang ${ }^{\mathrm{a}}$, Jian Shi ${ }^{\mathrm{a}}$, \\ Zhijun Zhang ${ }^{\mathrm{b}}$, Yong Chen ${ }^{\mathrm{a}, \mathrm{c}, \mathrm{d}, *}$
}

${ }^{a}$ École Normale Supérieure-PSL Research University, Département de Chimie, Sorbonne Universités - UPMC Univ Paris 06, CNRS UMR 8640 PASTEUR, 24, rue Lhomond, 75005 Paris, France

${ }^{b}$ Key Laboratory of Nano-Bio Interface, Division of Nanobiomedicine, Suzhou Institute of Nano-Tech and Nano-Bionics, Chinese Academy of Sciences, Suzhou, 215123, China

${ }^{c}$ Institute for Integrated Cell-Material Sciences, Kyoto University, Kyoto, 606-8507, Japan

${ }^{d}$ Institute for Interdisciplinary Research, Jianghan University, Wuhan, 430056, China

* Corresponding author. Email: yong.chen@ens.fr (Y. Chen) 


\begin{abstract}
Three dimensional (3D) printing techniques can be used for scaffold fabrication but the most of them are limited by resolution and material choice. To bypass these limitations, we developed an approach by combining conventional 3D printing and freeze-drying techniques to produce lattice-type backbone and embedding microporous structures. Polyethylene glycol diacrylate (PEGDA), a biocompatible and photosensitive pre-polymer, was chosen for 3D printing of the backbone, while gelatin was used for the formation of microporous structures. The fabricated PEGDA/gelatin scaffolds were used for culture and differentiation of neural progenitor cells (NPCs), showing infiltration of the cells and outgrowth of differentiated neurites. This strategy of combined use of 3D printing and freeze-drying techniques might be useful for scaffold fabrication in terms of easy design and easy material processing.
\end{abstract}

Key words: 3D printing, PEGDA, gelatin, microstructure, neuron differentiation 


\section{Introduction}

Three dimensional (3D) printing is an emerging technique for manufacturing in different fields such as automobile, aircraft, food, medical service, etc. [1-3]. In regenerative medicine, 3D printing also holds high potential for the fabrication of personalized scaffolds due to its unique advantages of easy design and fast processing [4-7]. Technology limitations, however, still lie ahead for printing complex structures at high resolution and high biocompatibility. To this regards, the efforts are directed to find printable biomaterials that have good stiffness but suited for cell culture and tissue formation [8].

Polyethylene glycol (PEG), for example, is nontoxic and non-immunogenic polymer, which is widely used in industrial manufacturing and medicine $[9,10]$. Once end capped with acrylate groups, PEG diacrylate (PEGDA) is formed which can be used for 3D printing. The stiffness of cross-linked PEGDA is also excellent [11, 12] but cells cannot adhere, remodel or proliferate in a PEGDA scaffold due to the inertial nature of PEG. On the other hand, scaffolds made by natural polymers such as collagen, gelatin, silk fibrin, chitosan, starch, alginate and hyaluronic can be used for cell culture and tissue formation but they are not suited for 3D printing. For example, microporous structures of gelatin can be obtained by lyophilizing or "freezing-drying" process, which can be used not only for cell attachment, proliferation and differentiation but also for implantation with low immunogenicity [13-17]. However, the fabricated microporous structures of gelatin have low stiffness and they cannot be produced by 3D printing [18].

In this work, we report a fabrication strategy of combined use of 3D printing and freezedrying techniques to bypass the above described difficulties (Fig. 1). First, a lattice type of PEGDA backbone of the scaffold is designed and produced by 3D printing. Then, gelatin gel is filled in the scaffold and freeze-dried to obtain sponge-like microporous structures of gelatin in the free-space of the PEGDA lattice. Afterward, the PEGDA/gelatin hybrid scaffold is used for culture and differentiation of neuron progenitor cells to prove the reliability of the method. Thus, the advantage of this method relies on solid freeform fabrication of the scaffold backbone by computer design and 3D printing and flexible integration of microporous structures by framed freeze-drying method. Our results show that the printed PEGDA scaffold is strong enough against mechanical stresses whereas the porous gelatin structures, supported by the PEGDA scaffold, have unchanged swellability and degradability after integration. Our results also show that the PEGDA/gelatin scaffold is excellent for cell attachment due to much increased biomaterial surface areas. Finally, we proved that the PEGDA/gelatin scaffolds were suited for culture and differentiation of neural progenitor cells 
(NPCs).

\section{Materials and methods}

\subsection{Materials}

A 3D Printer (ProJet 1200) and polyethylene glycol diacrylate (PEGDA) was purchased from 3D Systems, USA. Gelatin, N-(3-Dimethylaminopropyl)-N'-ethylcarbodiimide hydrochloride (EDAC), DAPI, Poly D-Lysine (PDL), and N-Hydroxysuccinimide (NHS) were from Sigma, France. Laminin, NPC growth medium and differentiating medium were purchased from StemCell, Inc., France. Anti- $\beta$ III tubulin antibody was purchased from Abcam, France.

\subsection{Fabrication of 3D PEGDA scaffold}

Lattice-type microstructures of polyethylene glycol diacrylate (PEGDA) were designed using software SolidWorks and produced by photopolymerization with the 3D printer and a PEGDA solution. PEGDA we used is in pure liquid form with its density, tensile strength and tensile modulus being $1.04 \mathrm{~g} / \mathrm{mL}, 30 \mathrm{MPa}$ and $1.7 \mathrm{GPa}$, respectively. After parameter setting, the 3D structure was produced, followed by immediate washing in isopropanol and postbaking with UV light.

\subsection{Preparation of PEGDA/gelatin scaffold}

The 3D PEGDA structure was immersed in a gelatin solution ( $8 \%$ in water), frozen and lyophilized for $24 \mathrm{~h}$. Afterwards gelatin was cross-linked in EDAC and NHS ethanol solution, followed by washing with ethanol and drying in vacuum.

\subsection{Morphology of PEGDA/gelatin scaffold}

The fabricated PEGDA/gelatin scaffolds were examined by scanning electron microscope (SEM) with an accelerating voltage of $10 \mathrm{kV}$. All samples were sputter coated with a $10 \mathrm{~nm}$ gold film before SEM observation.

\subsection{Swellability of PEGDA/gelatin scaffold}

The equilibrium swelling ratio $\left(\mathrm{Q}_{S}\right)$, which is a measure of swellability of hydrogel materials, was estimated by immersing the dried PEGDA/gelatin samples to swell in PBS at $37{ }^{\circ} \mathrm{C}$ for 7 days. $\mathrm{Q}_{S}$ was defined according to the following equation: 


$$
Q_{s}=\frac{W_{s}-W_{d}}{W_{d}}
$$

where Ws and Wd are the weights of the swollen sample and the dried sample, respectively [19].

\subsection{Degradability of PEGDA/gelatin scaffold}

The degradation ratio of scaffold was measured after 30 days incubation. Samples, in triplicate, were incubated in physiological saline solution with $0.01 \%$ trypsin at $37{ }^{\circ} \mathrm{C}$, respectively. The samples were weighed after being frozen and lyophilized. The degradation ratio $\left(\mathrm{R}_{d}\right)$ of scaffold was calculated according to the following equation:

$$
R_{d}=\frac{W_{0}-W_{30}}{W_{0}}
$$

where $\mathrm{W}_{0}$ and $\mathrm{W}_{30}$ represent the initial dried weight and the dried weight of the sample at day 30 , respectively.

2.7 Neural Progenitor Cell (NPC) extraction and culture in vitro

NPCs were obtained from the frontal cortex of unborn mouse after 14-day pregnancy. The obtained NPCs were cultured and expanded in NeuroCult ${ }^{\mathrm{TM}}$ Basal Medium (Mouse) with $10 \%$ NeuroCult ${ }^{\mathrm{TM}}$ Proliferation Supplement (Mouse), $20 \mathrm{ng} / \mathrm{mL}$ Epidermal Growth Factor (EGF, human) and $10 \mathrm{ng} / \mathrm{mL}$ basic Fibroblast Growth Factor (bFGF, human) under $37{ }^{\circ} \mathrm{C}$ and $5 \%$ $\mathrm{CO}_{2}$ in a humidified atmosphere incubator.

\subsection{Cell viability in PEGDA/gelatin scaffold}

Cell viability was measured by WST assay. WST is a chemical reagent which reacts with dehydrogenases, indicators of cell viability, in mitochondrion to form formazan. Cells with higher viability will produce more formazan. The resulted formazan can be quantified by microplate reader. Cell viability was calculated based on the absorbance intensity of formazan. Before cell culture, all PEGDA/gelatin scaffold samples were sterilized in $70 \%$ ethanol solution and coated with a layer of Laminin. NPCs were seeded in PEGDA/gelatin scaffolds at a density of $5 \times 10^{4}$ per well in 48 -well plate. After 1,3 and 5 days incubation, $20 \mu \mathrm{L}$ of WST solution was added into each well, followed by $1 \mathrm{~h}$ incubation. Cell proliferation was evaluated by cell viability based on the absorbance of each well at $450 \mathrm{~nm}$.

\subsection{Cell proliferation in PEGDA/gelatin scaffold}


NPCs were seeded in PEGDA/gelatin scaffolds at a density of $5 \times 10^{4}$ per well in 48 -well plate. After 1, 3 and 5 days incubation, $50 \mu \mathrm{L}$ of WST solution was added into each well, followed by $1 \mathrm{~h}$ incubation. Cell proliferation was evaluated by cell viability based on the absorbance of each well at $450 \mathrm{~nm}$.

\subsection{Calcien AM (CAM) and propidium iodide (PI) staining of live cells}

CAM/PI based Live/Dead cell viability assay was used to assess live and dead NPCs cultured on the scaffolds. $5 \times 10^{4}$ cells per well were seeded in Laminin coated PEGDA/gelatin scaffolds in 48-well plate. After 3 days of incubation, $20 \mathrm{ng} / \mathrm{L} \mathrm{CAM}$ and $10 \mathrm{ng} / \mathrm{L}$ PI were added and incubated for $15 \mathrm{~min}$. After being washed 3 times with PBS, the samples were detected by a Nikon Fluorescence Microscope.

\subsection{Neuron differentiation in PEGDA/gelatin scaffold}

NPCs were seeded in PEGDA/gelatin scaffolds at a density of $2 \times 10^{5}$ per well in 24 -well plate. After $24 \mathrm{~h}$ incubation, the culture medium was replaced by $1 \mathrm{~mL}$ neuron differentiating medium composed of Neurobasal Medium with $1 \%$ B27 and $1 \mathrm{mg} / \mathrm{L}$ L-Glutamic acid. After $3 \mathrm{~d}$ incubation, another $200 \mu \mathrm{L}$ of neuron differentiating medium was added into each well. After $5 \mathrm{~d}$ incubation, mature neurons can be obtained.

\subsection{Neuron staining and imaging}

After mature neurons were obtained, samples were washed with PBS (free of $\mathrm{Ca}^{2+}$ and $\mathrm{Mg}^{2+}$ ) three times, followed by $30 \mathrm{~min}$ fixation in $4 \%$ paraformaldehyde at $4{ }^{\circ} \mathrm{C}$. Samples were then permeated and blocked with commercially available solution for $1 \mathrm{~h}$. After primary antibody incubation overnight at $4{ }^{\circ} \mathrm{C}$ followed by washing for 3 times with immuno-staining wash buffer, samples were incubated with secondary antibody at room temperature for $2 \mathrm{~h}$ at $37^{\circ} \mathrm{C}$. At last samples were incubation with DAPI for nuclei staining for $30 \mathrm{~min}$. Fluorescence images were obtained by a Nikon A1 confocal laser scanning microscope.

\section{Results and discussion}

We first designed the PEGDA structure to be printed by SolidWorks, which consists of a 3D lattice with $200 \mu \mathrm{m}$ feature size and $800 \mu \mathrm{m}$ pitch size. The designed pattern was then loaded into the 3D printer. After parameter setting, the 3D structure was produced in 8 min for a scaffold of $1 \times 0.5 \times 0.25 \mathrm{~cm}^{3}$ in size (Fig. 2a). Afterward, the scaffold was washed in 
isopropanol and post-baked with UV light. As expected, this lattice type scaffold is strong enough to keep its shape but somehow elastic against the external force (Fig. 2b,c). Such a scaffold is not suited for cell culture because of the large pore size and the inert character of PEG. Fig.2d shows scanning electron microscope (SEM) image of the fabricated scaffold lattice. The growth rings of the PEGDA layers can be now seen on the surface of grid, due to layer-by-layer crosslinking of the 3D printing procedure.

The printed 3D PEGDA structure was used as backbone to support the formation of gelatin porous structures inside. The freeze-drying method is convenient and widely used in tissue engineering for the fabrication of microporous structures [20, 21]. Here, the PEGDA was immersed in a gelatin solution ( $8 \%$ in water), frozen and lyophilized. Afterward, gelatin was cross-linked in EDAC and NHS ethanol solution, followed by washing with ethanol and drying in vacuum. The as-synthesized PEGDA/gelatin scaffold was cut into two pieces and the cross section was characterized with SEM, as is shown in Fig. 2e. Now, the PEGDA/gelatin scaffold shows sponge-like gelatin microstructures which are embedded in the PEGDA grid. This sponge-like microstructure has high porosity due to the low content of gelatin, showing pore sizes in the range from $20 \mu \mathrm{m}$ to $100 \mu \mathrm{m}$ which are closed to cellular sizes (Fig. 2f). This highly porous structure will facilitate diffusion of nutrients and metabolic products and provide excellent 3D microenvironment for cells.

The swellability of PEGDA/gelatin scaffolds was investigated to determine the extent of crosslinking. The fabricated scaffold was immersed in PBS and weighed after 1, 3, and 7 days. The resulted swellability is shown in Fig. 3a. This scaffold can hold about 8 times of its dried weight within one day and tends to be saturated after 7 days, estimated to be 10 times of its dried weight. The high water-holding capacity is beneficial for tissue engineering, providing enough space for diffusion of nutrients and metabolism products as well as for cell growth, differentiation and interaction. Degradability is another factor to evaluate extent of crosslinking. A proper degradation time of the scaffold will not only ensure enough time for tissue treatment but also avoid long time retention in vivo. Scaffold with higher crosslinking ratio needs more time to degrade, while lower crosslinking ratio means shorter degradation time needed. To mimic the physiological condition in vivo, PEGDA/gelatin scaffold was immersed and incubated with PBS containing trypsin at $37^{\circ} \mathrm{C}$ with $5 \% \mathrm{CO}_{2}$ in incubator. After 10, 20, and 30 days incubation, scaffolds were rinsed with PBS, lyophilized overnight and weighed, as shown in Fig. 3b. The gelatin degraded, weighed about $45 \%$ of initial weight after 30 days, while PEGDA didn't and was deducted. This degradation rate is comparable to that of gelatin nanofiber reported before [22]. The degradation time is therefore enough for NPCs 
differentiation into neurons.

Biocompatibility is a prerequisite for applications of PEGDA/gelatin scaffolds in tissue engineering. We first studied the in vitro toxicity of PEGDA/gelatin scaffold by WST assay after NPCs cultured on scaffold for 24h. To make NPCs adhere to the scaffold, PEGDA/gelatin scaffold was coated with a layer of Laminin (NPCs will adhere on the Laminin coated area). The viability of NPCs was used to assess the cytotoxicity of scaffold toward NPCs cultured on it. For comparison, traditional tissue culture plate (TCP) was used as a control (100\% cell viability) and pure gelatin scaffold was used as another control. As shown in Fig. 4a, compared with TCP and pure gelatin scaffold, PEGDA/gelatin scaffold shows negligible toxicity toward NPCs, indicating its good biocompatibility.

The proliferation of NPCs on PEGDA/gelatin scaffold was then investigated, which is another factor to evaluate the biocompatibility of scaffold. NPCs were seeded in the PEGDA/gelatin scaffold for 1,3 , and 5 d culture, respectively. The proliferation of NPCs was determined by WST assay, as shown in Fig. 4b. Assuming the viability of NPCs of the control is $100 \%$ at day 1 , the viability of the NPCs cultured on PEGDA/gelatin scaffold are $11 \%, 52 \%$ and $85 \%$, at day 1, 3 and 5, respectively. After 5-day culture, NPCs are stained by CalceinAM (green)/PI (red) to show live and dead cells. The fluorescence spots shown in Fig. 4c have different brightness, suggesting that the NPCs were localized in different depths of the scaffold.

We further studied the differentiation of NPCs on PEGDA/gelatin scaffold. After 1-day culture, the culture medium was replaced by differentiation medium containing B27 and Glutamax acid. At day 5, differentiated NPCs could be observed. Fig. 5 shows the representative images of MAP-2 immuno-stained neurons after 7-day differentiation. As can be seen, each neuron has a spherical cell body with several thin cellular filaments extending for tens or hundreds of microns. These filaments tend to connect with each other (shown by white arrows) to form a large and probably 3D neuron network. We suggest that the PEGDA backbone played a key role to prohibit swelling induced large deformation of embedded gelatin foam, which should not be the case of the gelatin foam without backbone. Such a hierarchic 3D architecture should be in favor of neural network formation and their impulsion activities, thereby providing an alternative choice of the scaffolds for repairing injured nervous systems.

\section{Conclusion}

We have fabricated hierarchical scaffolds by 3D printing of PEGDA backbone and freeze- 
drying of gelatin gels. As expected, the printed PEGDA shows regular 3D grids with strong mechanical strength and sponge-like gelatin porous structures with good swellability and biodegradability. Cell culture has been studied, showing reasonable for attachment, proliferation and differentiation of NPCs in PEGDA/gelatin scaffolds. Interestingly, the porous structure of gelatin also provides structural cues for conduction of neural impulse. Finally, this combination strategy is also advantageous in terms of easy processing, scaling-up, and translating from one type of scaffolds to another.

\section{Acknowledgement}

This work was supported by the European Commission under contract No.604263 (Neuroscaffolds) and Agence de Recherche Nationale under contract No ANR-13-NANO0011-01 (Pillarcell) and ANR-12-RPIB-0015 (CardiacPatch). Z. Z and L. W. acknowledge support from NSFC (No. 51361130033) and MOST (No. 2014CB965003).

\section{Reference}

[1] M. Sugavaneswaran, G. Arumaikkannu, Modelling for randomly oriented multi material additive manufacturing component and its fabrication, Mater. Des. 54 (2014) 779-785. 
[2] B. Garrett, 3D printing: new economic paradigms and strategic shifts, Global Policy, 5 (2014) 70-75.

[3] D. Dimitrov, K. Schreve, N. De Beer, Advances in three dimensional printing-state of the art and future perspectives, J. New Generat. Sci. 4 (2006) p. 21-49.

[4] S.V. Murphy, A. Atala, 3D bioprinting of tissues and organs, Nat. Biotechnol. 32 (2014) 773-785.

[5] C.C. Chang, E.D. Boland, S.K. Williams, J.B. Hoying, Direct-write bioprinting three-dimensional biohybrid systems for future regenerative therapies, J. Biomed. Mater. Res. B Appl. Biomater. 98 (2011) 160-170.

[6] Y. Xu, X. Wang, Application of 3D Biomimetic Models in Drug Delivery and Regenerative Medicine, Curr. Pharm. Des. 21 (2015) 1618-1626.

[7] H.N. Chia, B.M. Wu, Recent advances in 3D printing of biomaterials, J. Biol. Eng. 9 (2015) 4.

[8] B. Seal, T. Otero, A. Panitch, Polymeric biomaterials for tissue and organ regeneration, Mater. Sci. Eng. R 34 (2001) 147-230.

[9] F.M. Veronese, A. Mero, The impact of PEGylation on biological therapies, BioDrugs 22 (2008) 315-329.

[10] K. Ueno, S. Nakamura, H. Shimotani, A. Ohtomo, N. Kimura, T. Nojima, H. Aoki, Y. Iwasa, M. Kawasaki, Electric-field-induced superconductivity in an insulator, Nat. Mater. 7 (2008) 855-858.

[11] V. Chan, J.H. Jeong, P. Bajaj, M. Collens, T. Saif, H. Kong, R. Bashir, Multi-material biofabrication of hydrogel cantilevers and actuators with stereolithography, Lab Chip 12 (2012) 88-98.

[12] M.P. Cuchiara, A.C. Allen, T.M. Chen, J.S. Miller, J.L. West, Multilayer microfluidic PEGDA hydrogels, Biomaterials 31 (2010) 5491-5497.

[13] J.B. Rose, S. Pacelli, A.J.E. Haj, H.S. Dua, A. Hopkinson, L.J. White, F.R. Rose, Gelatinbased materials in ocular tissue engineering, Materials 7 (2014) 3106-3135.

[14] Z. Guo, J. Xu, S. Ding, H. Li, C. Zhou, L. Li, In vitro evaluation of random and aligned polycaprolactone/gelatin fibers via eletrospinning for bone tissue engineering, J. Biomater. Sci. Polym. Ed. 26 (2015) 989-1001.

[15] M. Norouzi, I. Shabani, H.H. Ahvaz, M. Soleimani, PLGA/gelatin hybrid nanofibrous scaffolds encapsulating EGF for skin regeneration, J. Biomed. Mater. Res. A 103 (2014) 2225-2235.

[16] C.-H. Chuang, R.-Z. Lin, H.-W. Tien, Y.-C. Chu, Y.-C. Li, J.M. Melero-Martin, Y.-C. 
Chen, Enzymatic regulation of functional vascular networks using gelatin hydrogels, Acta Biomater. 19 (2015) 85-99.

[17] Y. Liu, M.B. Chan-Park, Hydrogel based on interpenetrating polymer networks of dextran and gelatin for vascular tissue engineering, Biomaterials, 30 (2009) 196-207.

[18] R. Dash, M. Foston, A.J. Ragauskas, Improving the mechanical and thermal properties of gelatin hydrogels cross-linked by cellulose nanowhiskers, Carbohyd. Polym. 91 (2013) 638645.

[19] K. Chawla, T.B. Yu, S.W. Liao, Z. Guan, Biodegradable and biocompatible synthetic saccharide- peptide hydrogels for three-dimensional stem cell culture, Biomacromolecules, 12 (2011) 560-567.

[20] H. Shen, Y. Ma, X. Liu, Z. Zhang, J. Dai, Directed osteogenic differentiation of mesenchymal stem cell in three-dimentional biodegradable methylcellulose-based scaffold, Colloid Surface B, 135 (2015) 332-338.

[21] X. Li, Z. Xiao, J. Han, L. Chen, H. Xiao, F. Ma, X. Hou, X. Li, J. Sun, W. Ding, Y. Zhao, B. Chen, J. Dai, Promotion of neuronal differentiation of neural progenitor cells by using EGFR antibody functionalized collagen scaffolds for spinal cord injury repair, Biomaterials, 34 (2013) 5107-5116.

[22] J. Fu, Y. Huang, G. Sui, X. Yang, Crosslinking and degradation of gelatin nanofiber membranes. J. B. Univ. Chem.Technol. 35 (2008) 58-62. 


\section{Figure caption}

Fig. 1 Fabrication and cell assay steps of PEDGA/gelatin scaffolds.

Fig. 2 Fabrication and characterization of PEGDA/gelatin scaffolds. a) Photograph of a typical PEGDA scaffold produced by 3D printing; b) and c) Deformation effects of PEGDA by pressing and pulling. Briefly, a centrifuge tube containing $50 \mathrm{~mL}$ water (total weight $63 \mathrm{~g}$ ) was used to: 1) press the PEGDA scaffold directly (Fig. 2 b) and 2) pull the PEGDA scaffold by hanging with strings (Fig. $2 \mathrm{c}$ ). The enlarged figures showed detailed shape of PEGDA scaffold when external forces applied, showing that the scaffolds exhibit negligible deformation; (d) SEM image of printed 3D PEGDA backbone; (e-f) SEM images of gelatin porous structure filled in the PEGDA scaffold.

Fig. 3 (a) Equilibrium swelling ratio of PEGDA/gelatin scaffold in PBS at $37{ }^{\circ} \mathrm{C}$. Samples were weighed at $1^{\text {st }}, 3^{\text {rd }}$ and $7^{\text {th }}$ day. (b) Biodegradability of PEGDA/gelatin scaffold in PBS at $37{ }^{\circ} \mathrm{C}$. Samples were weighed every 10 days.

Fig. 4 (a) Cell viability of NPCs cultured on PEGDA/gelatin and pure gelatin scaffold after 24 $\mathrm{h}$ incubation. Cells on tissue culture plate were used as a control (100\% cell viability). (b) Cell viability of NPCs cultured on PEGDA/gelatin scaffold for 1, 3 and 5 days. Tissue culture plate was used as a control (cell viability after 1-day culture is set as 100\%). (c) Fluorescence images of NPCs on PEGDA/gelatin scaffold after 5-day culture and Calcein-AM (green) and PI (red) staining.

Fig. 5 Fluorescent image of MAP-2 immuno-stained neurons (red) after 7-day differentiation, showing clearly spherical cell bodies and thin cellular filaments which are connected with each other (indicated by white arrows) to form a large network. 
Figure 1

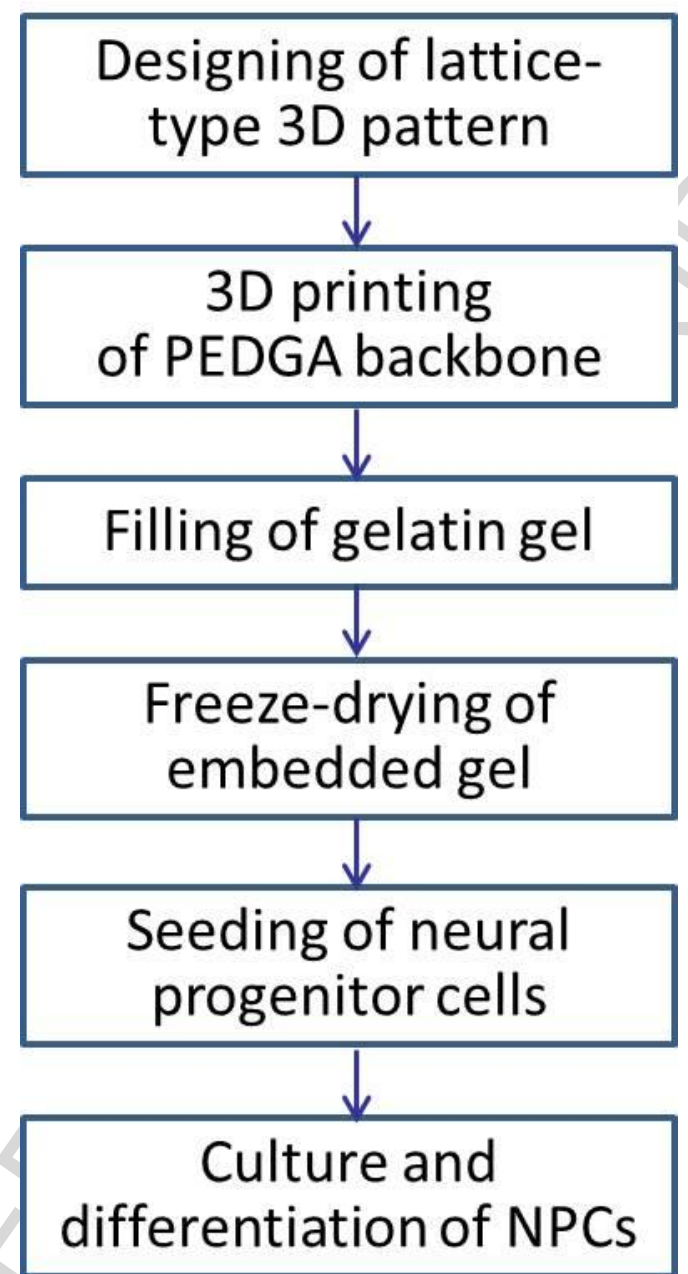


Figure 2
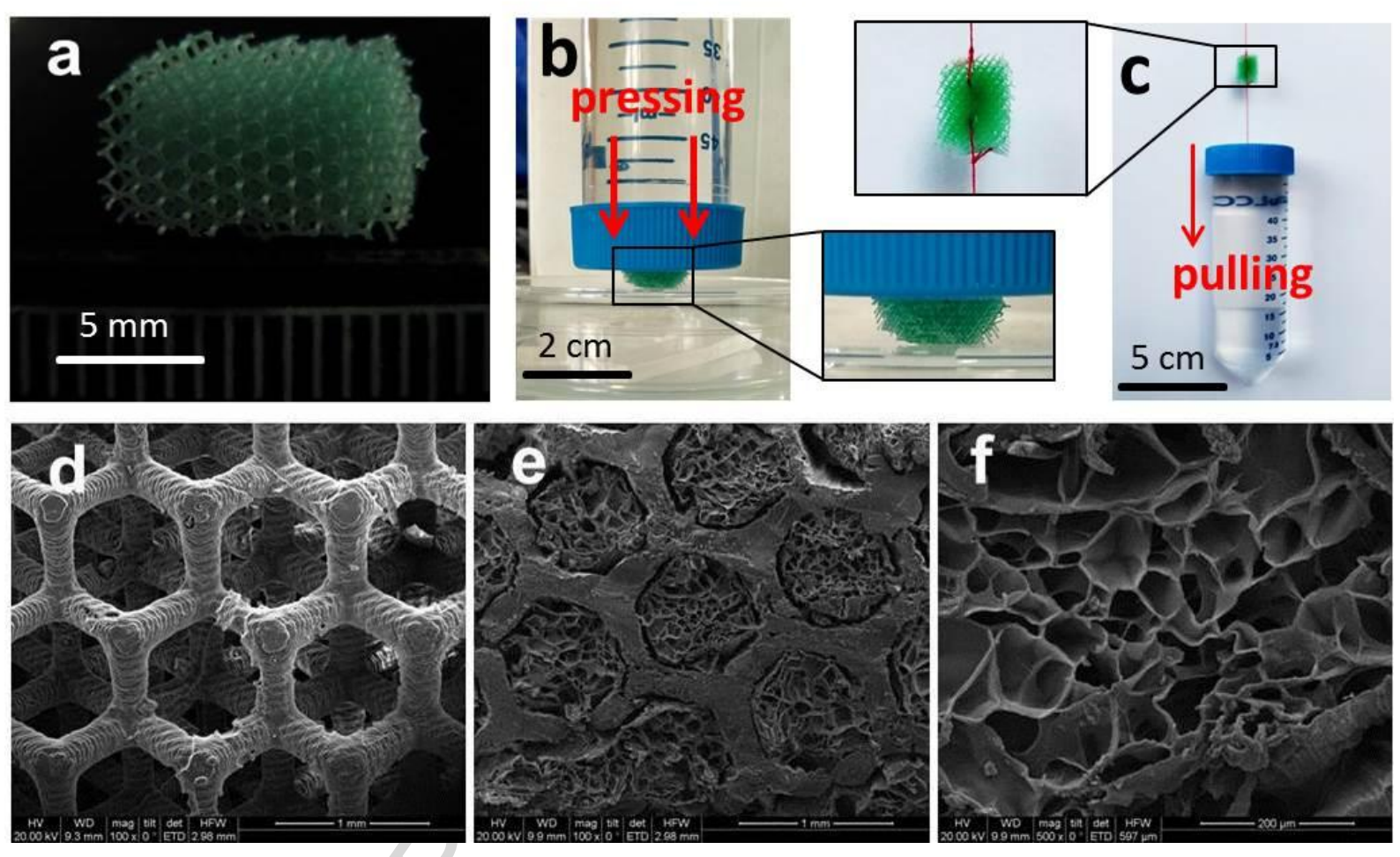
Figure 3
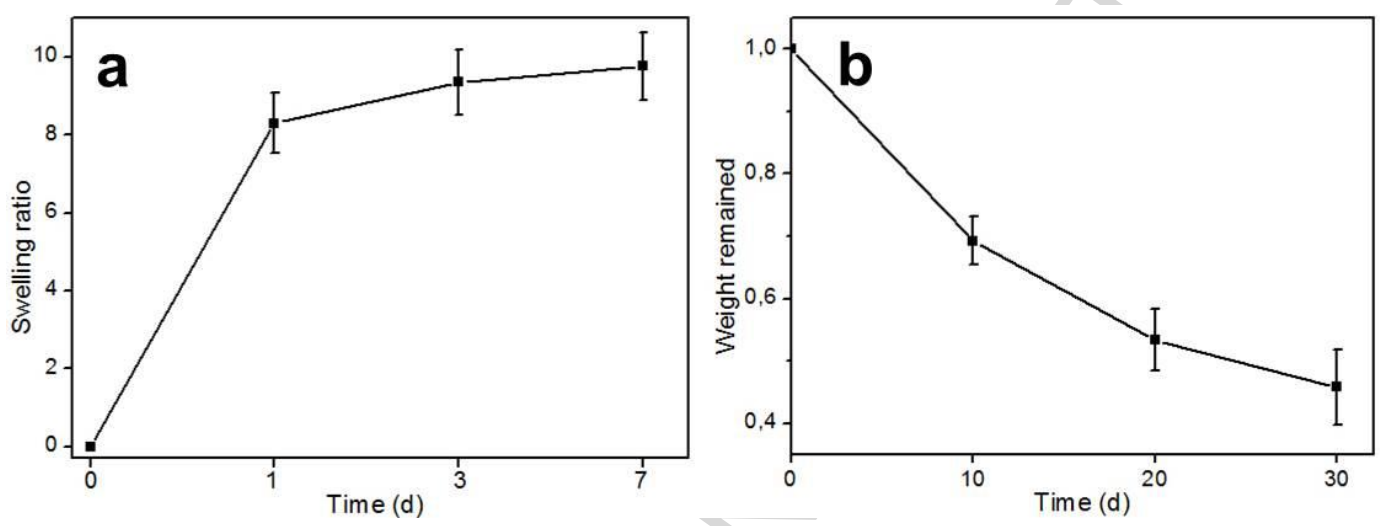
Figure 4
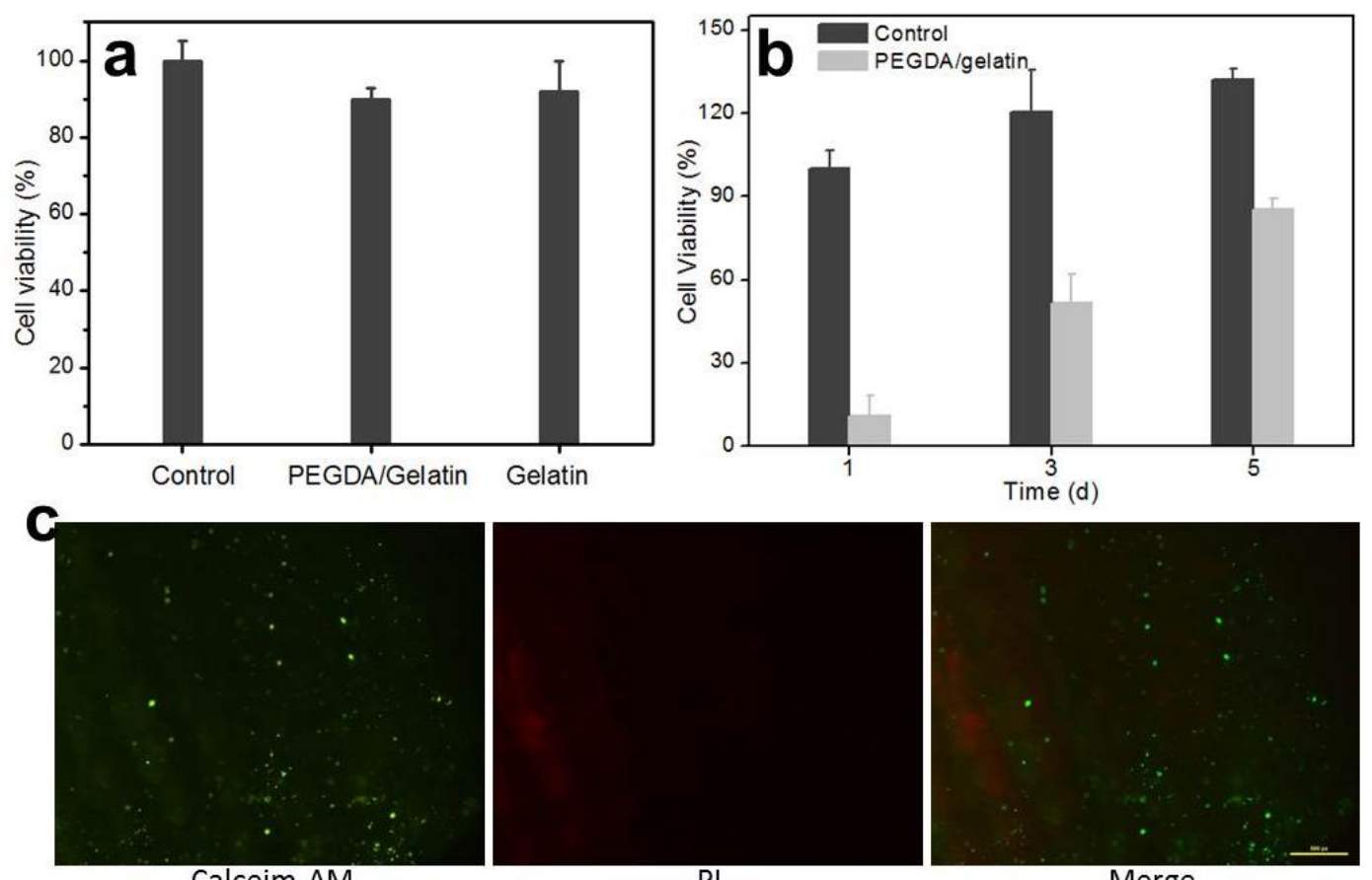

Calceim-AM

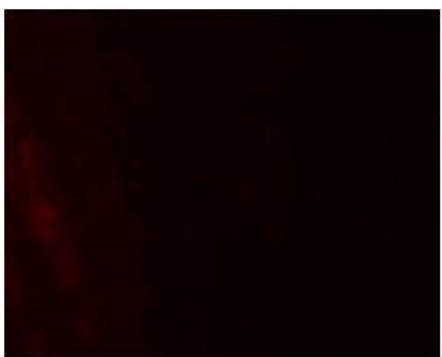

PI

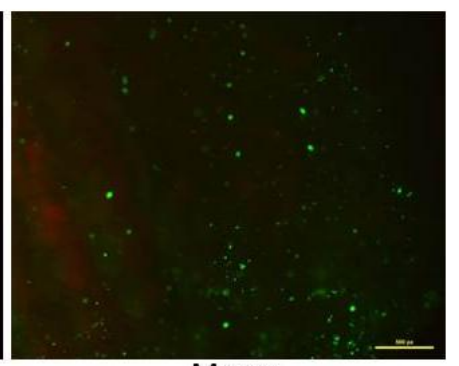

Merge 
Figure 5
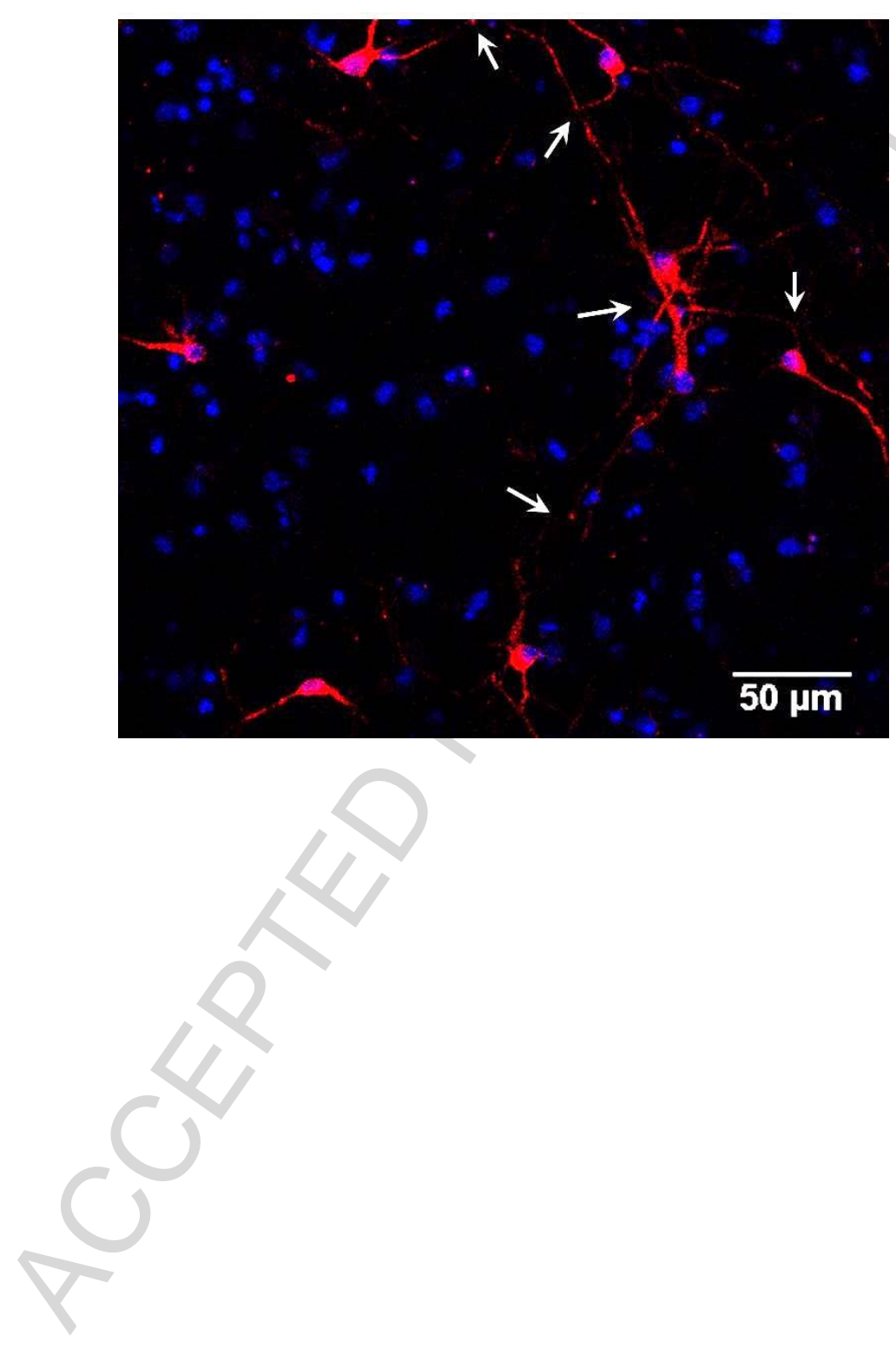
3D printed PEGDA Porous gelatin integrated
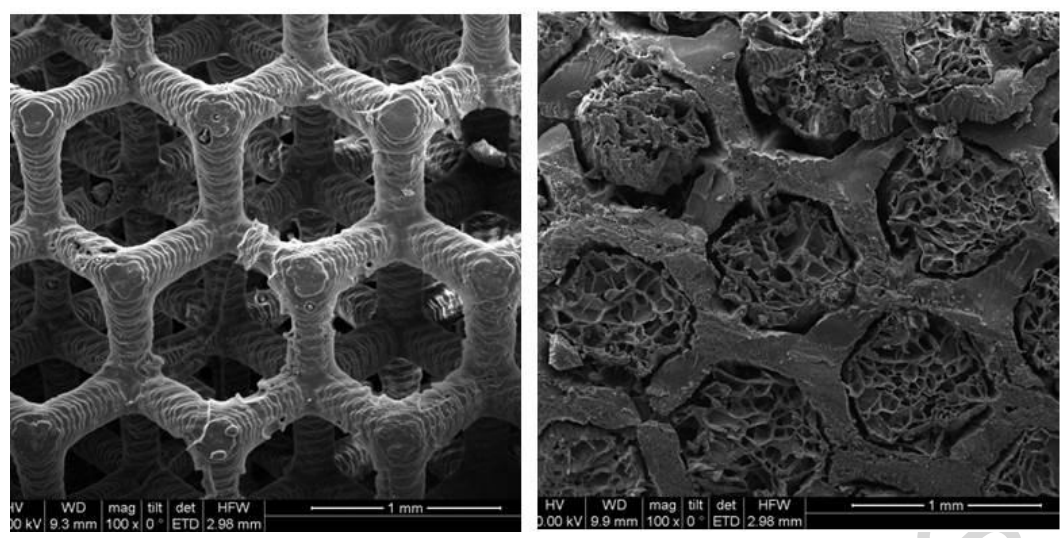

Scaffold with neurons

Graphical abstract

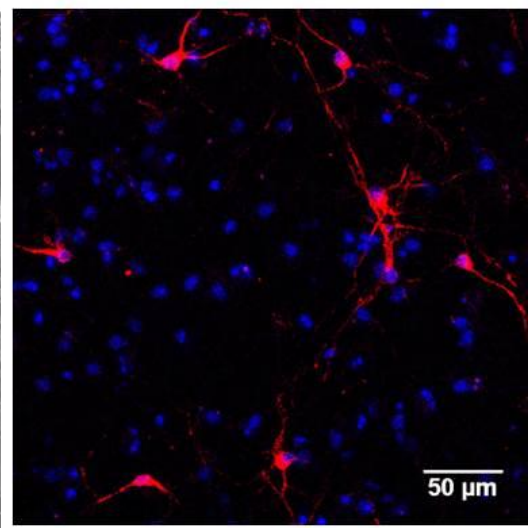




\section{Highlights}

1) 3D printing is used to produce a lattice-type backbone of PEGDA scaffold.

2) Microporous structures are obtained by freeze-drying of gelatin gel filled in the scaffold.

3) The PEGDA/gelatin hybrid scaffold is compatible to cell culture and tissue engineering.

4) The scaffold is used for differentiation of neuron progenitor cells. 